\title{
Inflammatory myofibroblastic tumor in the pancreatic neck: a rare case report and literature review
}

\author{
Zhe Liu, Guichen Li, Anjiang Gou, Zhihuan Xiao, Yuanhong Xu, Shaowei Song, Kejian Guo, Gang Ma \\ Department of Pancreatic-Biliary Surgery, First Hospital of China Medical University, Shenyang, China \\ Correspondence to: Dr. Gang Ma. Department of Pancreatic-Biliary Surgery, First Hospital of China Medical University, 155 North Nanjing St, \\ Shenyang 110001, China. Email: liuzhe4321@126.com.
}

\begin{abstract}
Inflammatory myofibroblastic tumor (IMT) is a rare disease of unknown etiology. It usually occurs in abdominal soft tissues and lung, and is extremely rare in the pancreas. IMT can occur in any part of a person at any age, however, it mostly affects children and young people. Its clinical manifestations are atypical, imaging examinations are not specific, and the differential diagnosis of pancreatic malignancies is difficult, making it easily misdiagnosed. Surgical resection is the preferred method of treatment for IMT. In this case report, we report a rare case of IMT in the neck of the pancreas and reviewed the relevant literature. In our case, the patient was a 57-year-old woman with an IMT in the neck of the pancreas. Abdominal pain was the only clinical symptom, and imaging features were not specific. She underwent surgery to remove the pancreatic mass, and the final diagnosis of IMT was based on histopathology and immunohistochemistry. After 6 months of regular follow-up, the patient had no complications or further incidents. The purpose is to emphasize the difficulty of the preoperative diagnosis of pancreatic IMT and the difficulty of distinguishing it from pancreatic malignancies. It is hoped that clinicians can gain a deeper understanding of pancreatic IMT through this case report.
\end{abstract}

Keywords: Inflammatory myofibroblastic tumor (IMT); diagnosis; neck of pancreas; abdominal pain; case report

Submitted Mar 29, 2021. Accepted for publication May 19, 2021.

doi: $10.21037 /$ gs-21-303

View this article at: http://dx.doi.org/10.21037/gs-21-303

\section{Introduction}

Inflammatory myofibroblastic tumor (IMT) is a rare mesenchymal tumor with recurrence potential. In 1990, the concept of IMT was proposed by Pettinato et al. (1), and it was officially named by the World Health Organization (WHO) in 2002. IMT is composed of differentiated myofibroblastic spindle cells, usually accompanied by a large number of plasma cells or lymphocytes (2). The cause of IMT is unknown. It may be related to the recombination of the ALK gene of the 2 p23 chromosome (3), or may be related to surgery, trauma, inflammation, Epstein-Barr virus $(4,5)$, and herpes simplex virus infection $(2,6,7)$, amongst other factors. It shows a wide anatomical distribution, most frequently arising in the abdominal soft tissues, followed by the lung, and unusual locations include somatic soft tissues, pancreas, liver, and the central nervous system (CNS).
The site of origin determines symptoms, and as many as one third of patients have a clinical syndrome, possibly cytokine-mediated, including fever, malaise, and weight loss, as well as laboratory abnormalities including anemia, thrombocytosis, and elevated erythrocyte sedimentation rate (8). Its clinical manifestations are atypical and imaging examinations are not specific. This makes preoperative diagnosis difficult, and it is likely to be misdiagnosed as a malignant tumor. Surgical resection is the preferred treatment. IMT of the pancreas is very rare. Herein, we report a case of IMT that occurred in the neck of the pancreas, discussed the relevant characteristics of pancreatic IMT, and reviewed the literature. To the best of our knowledge, IMT located in the neck of the pancreas has been rarely reported. We present the following article in accordance with the CARE reporting checklist (available at http://dx.doi.org/10.21037/gs-21-303). 


\section{Case presentation}

A 57-year-old female patient was admitted to the hospital on August 7, 2020, with intermittent abdominal pain for 2 months. The patient complained of pain in the right upper abdomen for no obvious reason 2 months ago, and the pain was obvious at night and relieved during the day. She underwent an abdominal ultrasound examination at the local hospital 2 weeks ago, and multiple gallbladder stones with inflammation and liver cysts were found. After conservative treatment, her symptoms eased slightly. She was seen at Anshan Central Hospital 1 week ago, and dynamic computed tomography (CT) of her abdomen revealed a space-occupying pancreatic lesion. During this time, she lost $3 \mathrm{~kg}$ in weight, without fever, vomiting, jaundice, melena, and other symptoms. Her blood pressure was $135 / 73 \mathrm{mmHg}$ and heart rate was $70 / \mathrm{min}$. There was no personal or family history. On physical examination, there was tenderness in the right upper abdomen, Murphy's sign was positive, and the patient's bowel sounds were normal. No special condition was found in other whole-body system examinations. Esophageal inflammation changes and atrophic gastritis were found in upper gastrointestinal endoscopy. Her WBC count was $4.95 \times 10^{9} / \mathrm{L}$, hemoglobin was $135 \mathrm{~g} / \mathrm{L}$, and her tumor markers (CEA: $4.19 \mathrm{ng} / \mathrm{mL}$, AFP: $2.07 \mathrm{ng} / \mathrm{mL}, \mathrm{CA}-125: 3.15 \mathrm{U} / \mathrm{mL}, \mathrm{CA}-19-9$ : $12.80 \mathrm{U} / \mathrm{mL}$ ) were all within the normal range.

CT showed that there was a circular low-density shadow with a diameter of $1.9 \mathrm{~cm}$ in the neck of the pancreas, and in contrast-enhanced CT, the mass was enhanced unevenly. It was highly suspected that this space-occupying lesion was a solid pseudopapillary tumor of the pancreas. There was a 1.4-cm-diameter round type of obvious enhancement in the right lobe of the liver, and it was unclear in the delayed phase. The gallbladder was not large, the gallbladder wall was thickened and strengthened, and multiple dense shadows were seen inside. CT suggested liver cysts and gallbladder stones with cholecystitis. Abdominal magnetic resonance imaging (MRI) showed that there was nodular long T1 and long T2 signal changes in the pancreas neck, the size was about $1.1 \mathrm{~cm} \times 1.9 \mathrm{~cm}$, and the enhancement showed uneven enhancement, which was lower than the surrounding pancreatic tissue. MRI showed a high possibility of solid pseudopapillary tumor of the pancreas. For the liver and gallbladder, MRI also had corresponding manifestations, and like CT, it suggested liver cysts and gallbladder stones.

At this point, the diagnosis of the patient was initially clear, but the nature of the pancreatic neck tumor was yet to be clarified. It remained to be determined whether it was a solid pseudopapillary tumor with high suspicion on CT and MRI imaging, other benign tumor of the pancreas, or a pancreatic malignant tumor. For diagnosis and treatment, laparoscopic surgery was performed on the patient. Firstly, under laparoscopy, it was found that the gallbladder showed chronic inflammation and adhered to the surroundings. The gallbladder was peeled along the gallbladder plate with an ultrasonic knife, and the gallbladder was removed. Later, after the pancreas was exposed, the tumor was found in the neck of the pancreas, leaning toward the head of the pancreas, about $2.0 \mathrm{~cm} \times 2.5 \mathrm{~cm}$ in size, with no infiltration in the surrounding area. During the operation, lymph nodes of groups 7, 8, and 9 were seen to be enlarged, and the frozen pathological results during the operation showed that the lymphatic tissue had proliferated, and no cancer was found. The patient underwent middle pancreatectomy. The tumor specimen was completely taken out during the operation, and the tumor envelope was intact (Figure $1 A, B$ ). After the specimen was cut open, it was found to be substantial, hard, and tough (Figure 1C). Intraoperative frozen pathology showed mesenchymal tumor, prone to aggressive fibromatosis. Immunohistochemical staining features suggested IMT. Two weeks after the operation, the patient was discharged from the hospital without complications.

All procedures performed in studies involving human participants were in accordance with the ethical standards of the institutional and/or national research committee(s) and with the Helsinki Declaration (as revised in 2013). Written informed consent was obtained from the patient. The study was approved by Ethics Committee of the First Affiliated Hospital of China Medical University

\section{Discussion}

IMT is a rare disease. In the past, IMT was also called plasma cell granuloma, inflammatory pseudotumor, inflammatory pseudotumor, inflammatory myofibrohistiocytic proliferation, omental-mesenteric myxoid hamartoma, and inflammatory fibrosarcoma. It was defined, in the fifth edition of the 2020 WHO classification of soft tissue and bone tumors, as a distinctive, rarely metastasizing neoplasm composed of myofibroblastic and fibroblastic spindle cells accompanied by an inflammatory infiltrate of plasma cells, lymphocytes, and/or eosinophils (9). There is generally no difference in the 


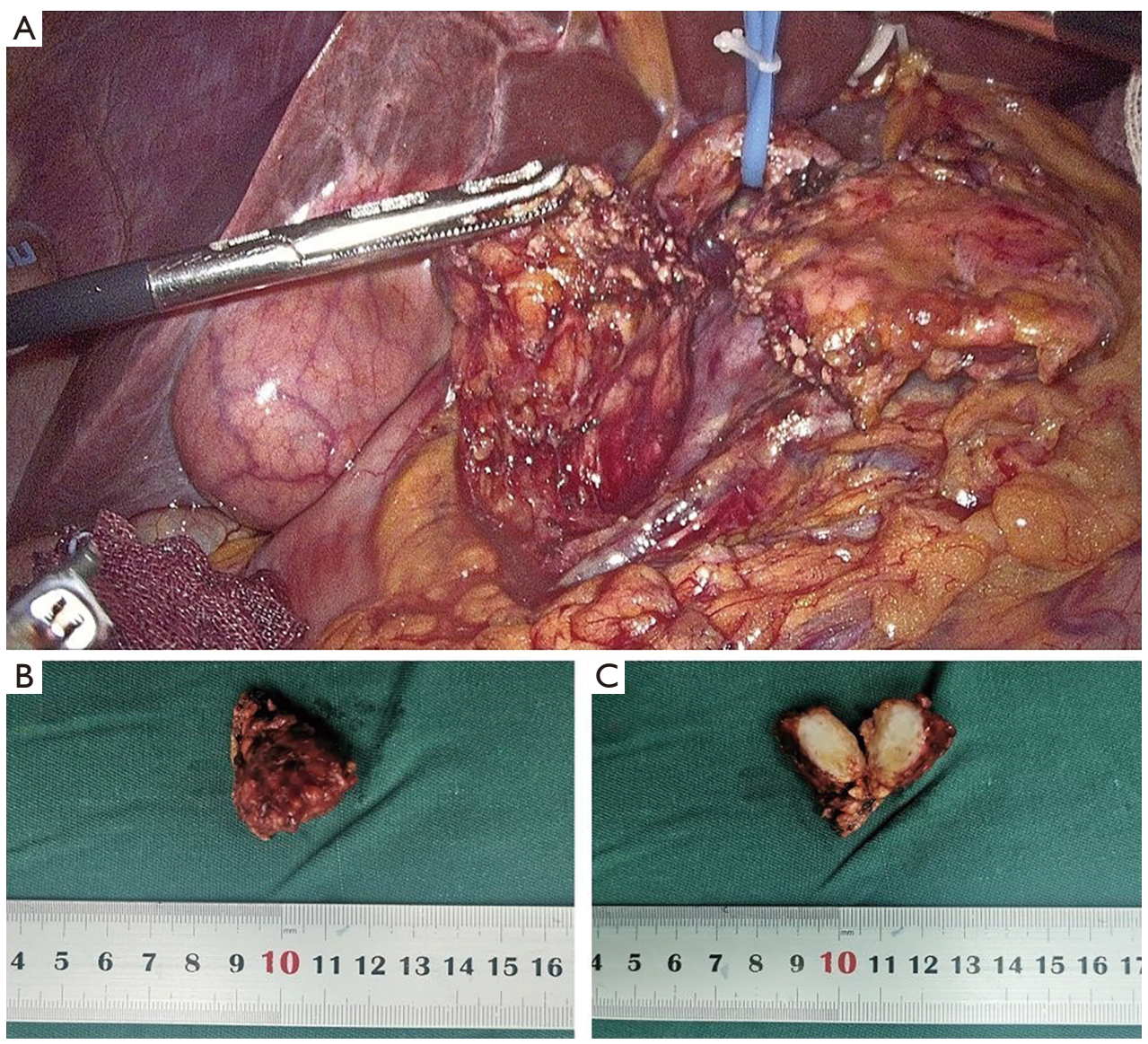

Figure 1 Photograph of the resected specimen. (A,B) The tumor specimen was completely taken out during the operation, and the tumor envelope was intact; (C) after the specimen was cut open, it was found to be substantial, hard and tough.

incidence of IMT in men and women, though females may have a slight predominance. IMT that occurs in the pancreas is quite rare, and while it can be located anywhere in the pancreas, it usually occurs in the head of the pancreas (10). In case reports of pancreatic IMT before $2004,60 \%$ of cases occurred in the head of the pancreas (10). In 2019, Matsubayashi et al. (11) searched PubMed for pancreatic IMT. After screening, there were only 27 cases of pancreatic IMT confirmed by histology, of which 20 cases were located in the head of the pancreas. IMT occurring in different locations of the pancreas may result in different clinical symptoms, but pancreatic IMT has no specific clinical symptoms and usually has no clinical symptoms or presents with general gastrointestinal symptoms such as abdominal pain, loss of appetite, nausea, and vomiting (10-12). This may be caused by the mass compressing the surrounding organs, such as the duodenum. In a few other cases, pancreatic IMT may manifest as anemia, fatigue, palpable mass, and diabetes, similar to pancreatic cancer and pancreatic neuroendocrine tumors (12). Interestingly, there was a case of pancreatic IMT reported showing extracolonic obstruction (13). There are also cases which present as acute pancreatitis $(14,15)$. In our case, IMT occurred in the neck of the pancreas, and the lump size was only $2.0 \mathrm{~cm} \times 2.5 \mathrm{~cm}$. Abdominal pain was the only clinical symptom of the patient. However, the patient was found to have gallstones and chronic cholecystitis, the right upper abdomen was tender, and Murphy's sign was positive. Therefore, abdominal pain seems to be caused by cholecystitis, but abdominal pain may not be related to pancreatic IMT.

The cause of IMT is unknown, and IMT is genetically heterogeneous. In $50-60 \%$ of IMT cases in children and young adults, there is a clonal cytogenetic rearrangement of the tumor, involving the $2 \mathrm{p} 23$ chromosome band, that fuses the 3' kinase region of the ALK gene with various partner genes. It is a rare mesenchymal tumor with the potential 


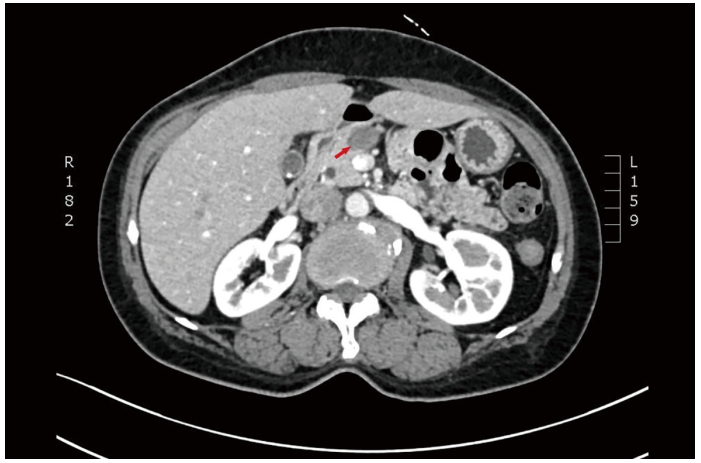

Figure 2 Enhanced abdominal CT revealed a circular low-density mass. A circular low-density shadow with a diameter of $1.9 \mathrm{~cm}$ in the neck of pancreas (pointed by the red arrow).

for recurrence, with intermediate biological behavior (lowgrade malignancy). The spindle fibroblastic-myofibroblastic cells and inflammatory cells form three basic histological patterns (9), including a myxoid pattern, a hypercellular pattern, and a hypocellular fibrous pattern.

Preoperative diagnosis of IMT is difficult because of its rarity and histological diversity, as well as the lack of specific clinical manifestations and imaging features. At present, there is no biomarker that can help the early diagnosis of IMT. Some pancreatic IMT imaging features are strikingly similar to pancreatic cancer, and most cases mimic malignancies (11), making it difficult to diagnose correctly. At present, CT diagnosis of pancreatic IMT has not been established by a standard or expert consensus. However, the CT of pancreatic IMT usually presents as low-density lesions. After enhancement, it shows uneven delayed enhancement, that is, early enhancement does not enhance or has mild enhancement, and late enhancement further enhances to moderate enhancement. However, in some cases, enhanced CT showed no enhanced mass, suspected to be ductal adenocarcinoma, and finally pathologically confirmed as pancreatic IMT (16). Therefore, the exact enhanced CT diagnostic criteria may be difficult for IMT. MRI of pancreatic IMT usually shows uneven low signal on T1WI, and equal, slightly high, and high signal on T2WI. Enhanced MRI shows uneven enhancement. However, there are also case reports showing low signal on both T1WI and T2WI (17). This may be related to the pathological classification of IMT, in that the greater the proportion of intratumoral stromal edema and mucus collagen degeneration, the higher the signal, and the greater the proportion of spindle cells, the lower the signal.
CT can accurately display the blood supply and anatomical characteristics of the lesion, while MRI can help evaluate the composition of the lesion and predict the pathological classification. If an unevenly enhanced soft tissue mass with infringement of adjacent tissue structures and unclear borders is found on the CT image, the possibility of IMT should be considered when diagnosis and differential diagnosis of the tumor at this site are involved. At the same time, ultrasound and CT-guided biopsy is helpful for the diagnosis of IMT, but this diagnosis method is rarely available in clinical practice.

The histological diagnosis of biopsy or intraoperative frozen sections is very difficult, and it is of little help for the diagnosis of IMT due to the vast inflammatory infiltration of the lesion (18). Pancreatic IMT must be differentiated from lymphoma, fibrosarcoma, chronic pancreatitis, and pancreatic cancer (19). The final diagnosis depends on histopathology and immunohistochemical examination $(10,20)$. The WHO's 2020 essential diagnostic criteria for IMT are as follows: (I) loose or compact fascicles of spindle cells with a prominent inflammatory infiltrate and a variable fibrous or myxoid stroma; (II) expression of ALK (seen in as many as $60 \%$ of cases). The desirable diagnostic criterion is ALK or other gene rearrangements (in selected cases). In our case, the CT image showed that the pancreatic neck had a circular low-density shadow with a diameter of $1.9 \mathrm{~cm}$, which was unevenly enhanced after enhancement (Figure 2), but the imaging doctor's report suggested that the diagnosis was likely to be solid pseudopapillary tumor. However, intraoperative frozen sections suggested invasive fibromatosis. The results were confirmed by pathology and immunohistochemistry, and the final diagnosis was IMT. Evidently, the whole disease diagnostic process was complicated, and highlights the difficulty of preoperative diagnosis of pancreatic IMT.

Surgical resection is the first choice to obtain a clear diagnosis and relieve symptoms, and it is also the only effective method for the treatment of pancreatic IMT. The clinical course of IMT is usually benign, and the tumor's biological potential is low. Therefore, the treatment of pancreatic IMT is different from the treatment of pancreatic cancer, radiotherapy and chemotherapy are rarely used in the treatment of IMT, and the benefits of chemotherapy and radiotherapy remain to be proven (21). The efficacy of chemotherapy drugs is still controversial (22-24). For highly invasive IMT, the necessity and specific efficacy of radiotherapy and chemotherapy require more large-scale studies to confirm. In addition, there were reports that 

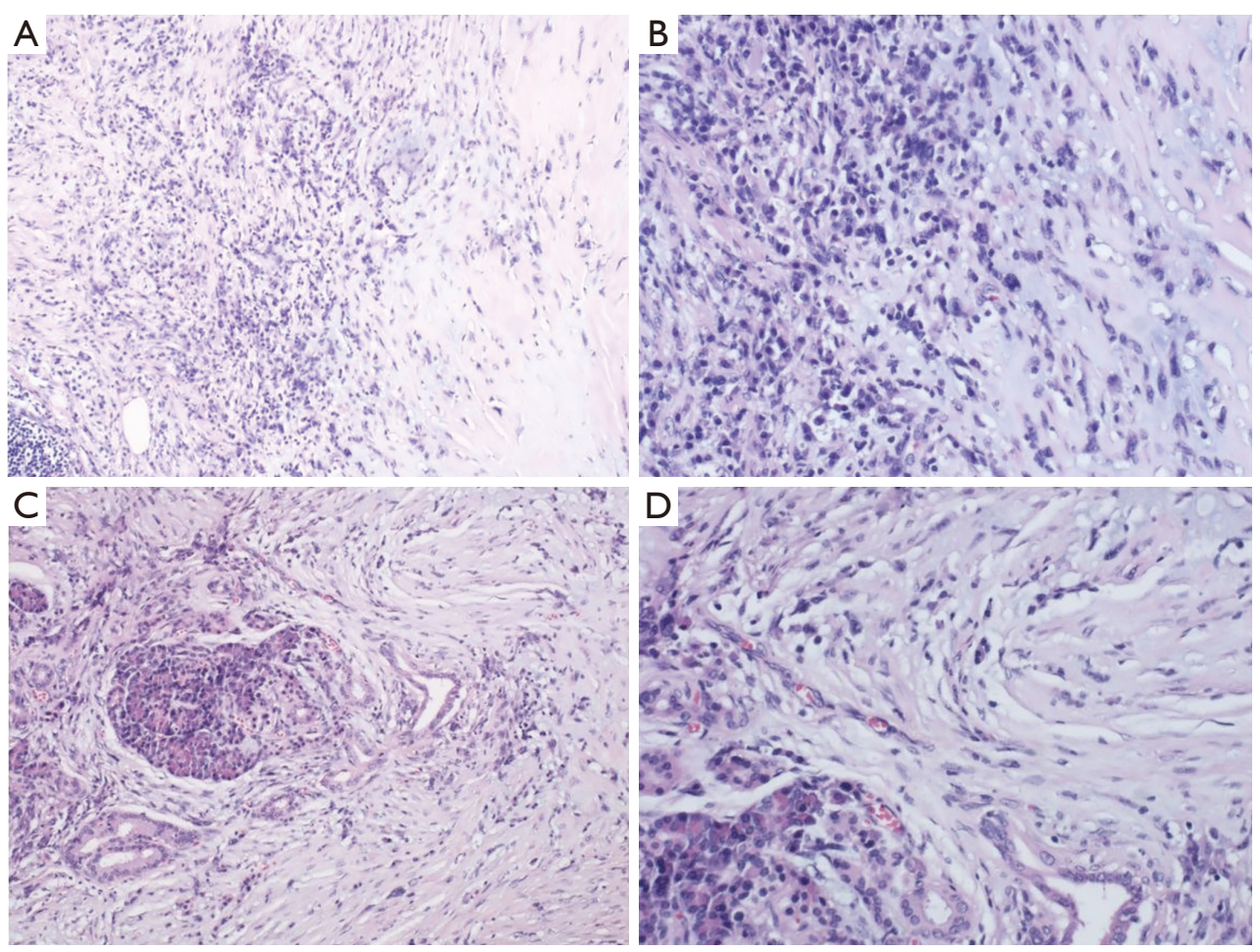

Figure 3 Photomicrograph of hematoxylin and eosin staining of the lesion. (A,B) The tumor cells are distributed in bundles or sheets, the cells are fusiform or epithelioid, the nucleus is oval or polygonal, nucleoli are visible, mitotic images are rare, and a lot of inflammatory cell infiltration is seen in the background, mainly plasma cells and lymphocytes. Local stroma with hyaline degeneration (magnification, $\times 100$, $\times 200$ respectively); (C,D) Tumor cells have unclear borders, infiltrate and grow into the surrounding pancreatic tissue, and the pancreatic ducts and pancreatic islets are compressed and deformed (magnification, $\times 100, \times 200$ respectively).

steroids and non-steroidal anti-inflammatory drugs can be used to treat IMT $(25,26)$. IMT may have local recurrence, but local invasive or malignant lesions with distant metastasis are rare (21). Incomplete resection, such as adhesion of important structures and multifocality, may be an important cause of IMT recurrence (8). IMT that occurs in the abdomen or retroperitoneum has a tendency to be more aggressive, and is more prone to multiple recurrences and distant metastases (21). A study in 2007 showed that ALK-negative IMT may be more likely to metastasize (3).

Typical myofibroblasts have a vesicular nucleus, 1-3 small nuclei, and an eosinophilic cytoplasm, and sometimes appear as ganglion-like nuclei. Necrosis is not common. When malignant transformation occurs, the uniform spindle cells transform into atypical polygonal cells or full cells, the nucleus is bubble-like, the nucleolus is prominent, the proliferation activity is enhanced, and tumor necrosis occurs (8). The mitotic phase and atypia of spindle cells in tumors are closely related to the invasion, metastasis, and recurrence of IMT. If there are many spindle-shaped nuclei in the tumor, it can easily be misdiagnosed as sarcoma or malignant fibrous histiocytoma. If the number of inflammatory cells in the tumor is abnormally increased, it can easily be confused with lymphoma. In our case, we could see local stroma with hyaline degeneration, and found that the typical spindle cells were infiltrated by a large number of inflammatory cells, mainly plasma cells and lymphocytes (Figure 3A,B). Furthermore, tumor cells had grown into surrounding pancreatic tissue (Figure $3 C, D$ ). On immunohistochemistry, the tumor was found to be positive for Vimentin (Figure $4 A$ ), smooth muscle actin (SMA) (Figure 4B), and CD34 (Figure 4C), but negative for $\beta$-catenin (Figure $4 D$ ). Some tumor cells had positive expression of Bcl-2 (Figure 4E), and the expression of Ki67 in tumor cells was about 5\% (Figure 4F). According to the results of histology and immunohistochemistry, we obtained a final diagnosis of pancreatic IMT. The prognosis of IMT is generally good. In the long-term follow-up of 

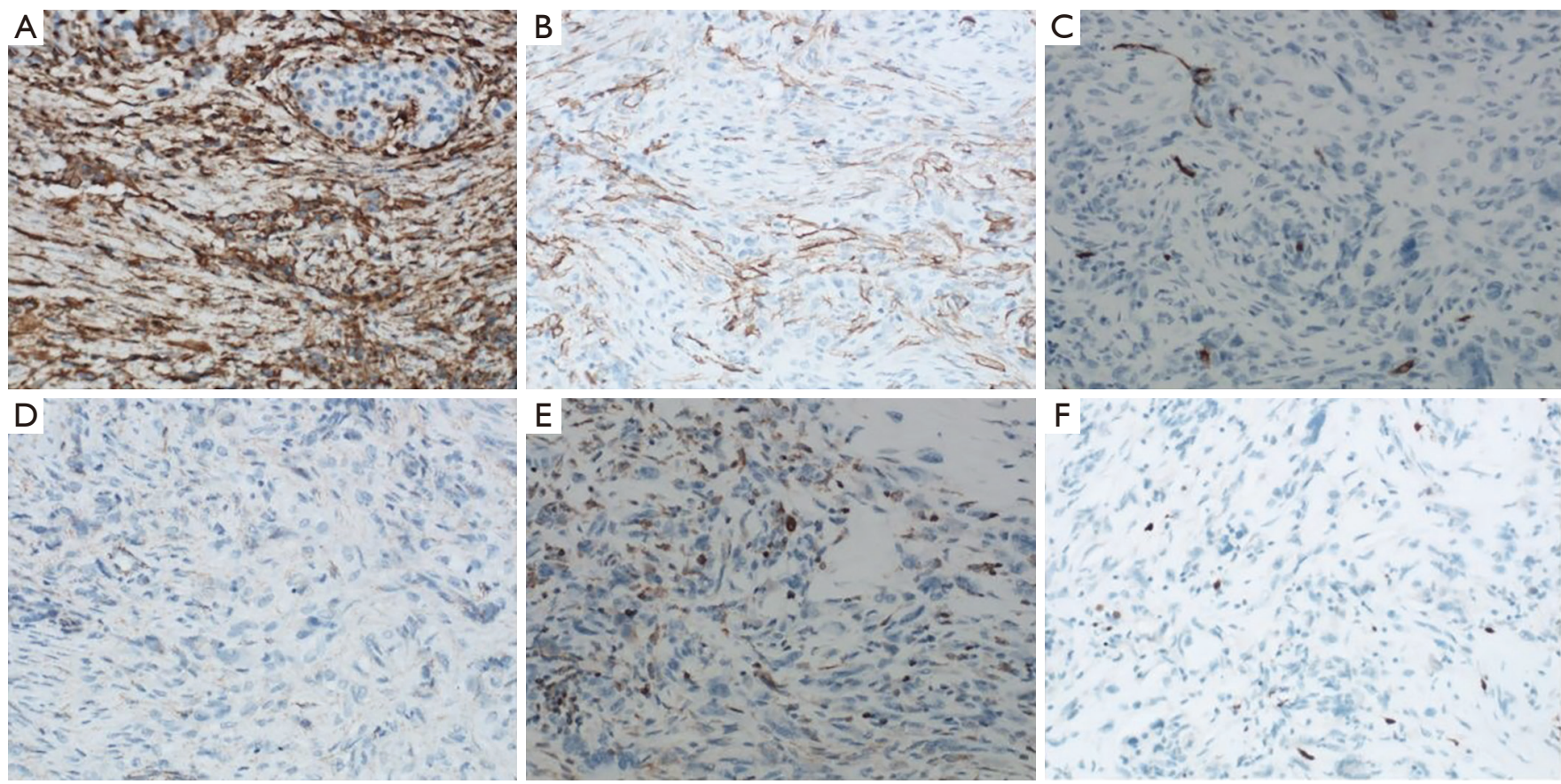

Figure 4 Photomicrograph of immunohistochemistry staining of the lesion. (A) Vimentin positive expression in tumor cells, negative expression in internal control pancreatic islet tissue. (B) Tumor cells were positive for smooth muscle actin. Smooth muscle of blood vessels acted as an internal positive control. (C) CD34 positive expression in tumor cells, positive expression in internal control blood vessels. (D) Tumor cells were immunonegative for $\beta$-catenin. (E) There are some tumor cells express Bcl-2 positively. (F) The expression of Ki67 in tumor cells is about $5 \%$. Magnification, $\times 200$.

pancreatic IMT, complications rarely occur. During the 6-month follow-up of this case, the patient had no other complications.

\section{Conclusions}

In summary, this case reports a rare case of IMT in the neck of the pancreas. The patient was 57 years old. She only had abdominal pain symptoms and non-specific imaging findings. Even professional imaging physicians made a diagnosis of solid pseudopapillary tumor. Intraoperative rapid frozen sections also failed to accurately diagnose, and the diagnosis ultimately depends on surgical resection, postoperative histopathological examination, and immunohistochemistry. Complete surgical resection is the first choice method to treat IMT. This case highlights the difficulty of preoperative diagnosis of pancreatic IMT, and emphasizes the difficulty of distinguishing it from pancreatic malignancies, even with multimodal investigation. As mentioned previously, preoperative diagnosis is usually incorrect, and most patients usually require surgical exploration and resection to confirm an accurate diagnosis. We hope that through this case report, clinicians can have a deeper understanding of pancreatic IMT.

\section{Acknowledgments}

Funding: Liaoning Provincial Department of Education Science Research Project (No. L2014299) and National Natural Science Foundation of China (No. 81572360).

\section{Footnote}

Reporting Checklist: The authors have completed the CARE reporting checklist. Available at http://dx.doi.org/10.21037/ gs-21-303

Conflicts of Interest: All authors have completed the ICMJE uniform disclosure form (available at http://dx.doi. org/10.21037/gs-21-303). All authors report funding from Liaoning Provincial Department of Education Science Research Project (No. L2014299) and National Natural 
Science Foundation of China (No. 81572360). The authors have no other conflicts of interest to declare.

Ethical Statement: The authors are accountable for all aspects of the work in ensuring that questions related to the accuracy or integrity of any part of the work are appropriately investigated and resolved. All procedures performed in studies involving human participants were in accordance with the ethical standards of the institutional and/or national research committee(s) and with the Helsinki Declaration (as revised in 2013). Written informed consent was obtained from the patient. The study was approved by Ethics Committee of the First Affiliated Hospital of China Medical University.

Open Access Statement: This is an Open Access article distributed in accordance with the Creative Commons Attribution-NonCommercial-NoDerivs 4.0 International License (CC BY-NC-ND 4.0), which permits the noncommercial replication and distribution of the article with the strict proviso that no changes or edits are made and the original work is properly cited (including links to both the formal publication through the relevant DOI and the license). See: https://creativecommons.org/licenses/by-nc-nd/4.0/.

\section{References}

1. Pettinato G, Manivel JC, De Rosa N, et al. Inflammatory Myofibroblastic Tumor (plasma cell granuloma): Clinicopathologic Study of 20 Cases with Immunohistochemical and Ultrastructural Observations. Am J Clin Pathol 1990;94:538-46.

2. Gómez-Román JJ, Ocejo-Vinyals G, Sánchez-Velasco P, et al. Presence of Human Herpesvirus-8 DNA Sequences and Overexpression of Human IL-6 and Cyclin D1 in Inflammatory Myofibroblastic Tumor (Inflammatory Pseudotumor). Lab Invest 2000;80:1121-6.

3. Coffin CM, Hornick JL, Fletcher CDM. Inflammatory Myofibroblastic Tumor: Comparison of Clinicopathologic, Histologic, and Immunohistochemical Features Including ALK Expression in Atypical and Aggressive Cases. Am J Surg Pathol 2007;31:509-20.

4. Arber DA, Kamel OW, van de Rijn M, et al. Frequent presence of the epstein-barr virus in inflammatory pseudotumor. Hum Pathol 1995;26:1093-8.

5. Lewis JT, Gaffney RL, Casey MB, et al. Inflammatory Pseudotumor of the Spleen Associated With a Clonal Epstein-Barr Virus Genome: Case Report and Review of the Literature. Am J Clin Pathol 2003;120:56-61.

6. Gómez-Román JJ, Sánchez-Velasco P, Ocejo-Vinyals G, et al. Human Herpesvirus-8 Genes Are Expressed in Pulmonary Inflammatory Myofibroblastic Tumor (Inflammatory Pseudotumor). Am J Surg Pathol 2001;25:624-9.

7. Slavotinek JP, Bourne AJ, Sage MR, et al. Inflammatory pseudotumour of the pancreas in a child. Pediatr Radiol 2000;30:801-3.

8. Coffin CM, Watterson J, Priest JR, et al. Extrapulmonary Inflammatory Myofibroblastic Tumor (Inflammatory Pseudotumor) A Clinicopathologic and Immunohistochemical Study of 84 Cases. Am J Surg Pathol 1995;19:859-72.

9. Board WCoTE. Soft Tissue and Bone Tumours. International Agency for Research on Cancer; 2020. Available online: https://publications.iarc.fr/Book-AndReport-Series/Who-Classification-Of-Tumours/SoftTissue-And-Bone-Tumours-2020

10. Pungpapong S, Geiger XJ, Raimondo MJJ. Inflammatory myofibroblastic tumor presenting as a pancreatic mass: a case report and review of the literature. JOP 2004;5:360-7.

11. Matsubayashi H, Uesaka K, Sasaki K, et al. A Pancreatic Inflammatory Myofibroblastic Tumor with Spontaneous Remission: A Case Report with a Literature Review. Diagnostics (Basel) 2019;9:150.

12. Gleason BC, Hornick JL. Inflammatory myofibroblastic tumours: where are we now? J Clin Pathol 2008;61:428-37.

13. İflazoğlu N, Kozan SK, Biri T, et al. Pancreatic inflammatory myofibroblastic tumor presenting with extracolonic obstruction. Turk J Surg 2020;36:233-7.

14. Panda D, Mukhopadhyay D, Datta C, et al. Inflammatory Myofibroblastic Tumor Arising in the Pancreatic Head: a Rare Case Report. Indian J Surg 2015;77:538-40.

15. Battal M, Kartal K, Tuncel D, et al. Inflammatory myofibroblastic pancreas tumor: a case report. Clin Case Rep 2016;4:1122-4.

16. Yamamoto $\mathrm{H}$, Watanabe $\mathrm{K}$, Nagata $M$, et al. Inflammatory myofibroblastic tumor (IMT) of the pancreas. J Hepatobiliary Pancreat Surg 2002;9:116-9.

17. Dulundu E, Sugawara Y, Makuuchi M. Inflammatory myofibroblastic tumor of the pancreas--a case report. Biosci Trends 2007;1:167-9.

18. Walsh SV, Evangelista F, Khettry U. Inflammatory Myofibroblastic Tumor of the Pancreaticobiliary Region: Morphologic and Immunocytochemical Study of Three Cases. Am J Surg Pathol 1998;22:412-8.

19. Meis JM, Enzinger FM. Inflammatory Fibrosarcoma of 
the Mesentery and Retroperitoneum: A Tumor Closely Simulating Inflammatory Pseudotumor. Am J Surg Pathol 1991;15:1146-56.

20. Sim A, Lee MW, Nguyen GK. Inflammatory myofibroblastic tumour of the pancreas. Can J Surg 2008;51:E23-4.

21. Difiore JW, Goldblum JR. Inflammatory myofibroblastic tumor of the small intestine. J Am Coll Surg 2002;194:502-6.

22. Jacob SV, Reith JD, Kojima AY, et al. An Unusual Case of Systemic Inflammatory Myofibroblastic Tumor with Successful Treatment with ALK-Inhibitor. Case Rep Pathol 2014;2014:470340.

23. Maruyama Y, Fukushima T, Gomi D, et al. Relapsed and unresectable inflammatory myofibroblastic tumor

Cite this article as: Liu Z, Li G, Gou A, Xiao Z, Xu Y, Song S, Guo K, Ma G. Inflammatory myofibroblastic tumor in the pancreatic neck: a rare case report and literature review. Gland Surg 2021;10(5):1832-1839. doi: 10.21037/gs-21-303 responded to chemotherapy: A case report and review of the literature. Mol Clin Oncol 2017;7:521-4.

24. Poves I, Alonso S, Jimeno M, et al. Retroperitoneal inflammatory pseudotumor presenting as a pancreatic mass. JOP 2012;13:308-11.

25. Doski JJ, Priebe CJ Jr, Driessnack M, et al. Corticosteroids in the management of unresected plasma cell granuloma (inflammatory pseudotumor) of the lung. J Pediatr Surg 1991;26:1064-6.

26. Ding D, Bu X, Tian F. Inflammatory myofibroblastic tumor in the head of the pancreas with anorexia and vomiting in a 69-year-old man: A case report. Oncol Lett 2016;12:1546-50.

(English Language Editor: C. Betlazar-Maseh) 